\title{
Time Series Modeling of Guinea Fowls Production in Kenya Using the ARIMA and ARFIMA Models
}

\author{
Cecilia Mbithe Titus*, Anthony Wanjoya, Thomas Mageto \\ Department of Statistics and Actuarial Science, Jomo Kenyatta University of Agriculture and Technology, Nairobi, Kenya \\ Email address: \\ cessm75@gmail.com (C. M. Titus), awanjoya@gmail.com (A. Wanjoya),tttmageto@gmail.com (T. Mageto) \\ ${ }^{*}$ Corresponding author
}

\section{To cite this article:}

Cecilia Mbithe Titus, Anthony Wanjoya, Thomas Mageto. Time Series Modeling of Guinea Fowls Production in Kenya Using the ARIMA and ARFIMA Models. International Journal of Data Science and Analysis. Vol. 7, No. 1, 2021, pp. 1-7. doi: 10.11648/j.ijdsa.20210701.11

Received: January 26, 2021; Accepted: February 6, 2021; Published: February 10, 2021

\begin{abstract}
Commercial farming of Guinea Fowls is at its infant stages and is generating a lot of interest for farmers in Kenya. This, coupled with an increased demand for poultry products in the Kenyan market in the recent past, calls for the rearing of the guinea fowls which are birds reared for meat and partly for eggs. In order to have an efficient production of poultry products for this type of poultry farming, there is need for an efficient modeling using sound statistical methodologies. It's in this regard that the study modeled Guinea Fowl production in Kenya using the Univariate Auto-Regressive Integrated Moving Average (ARIMA) and the Auto-Regressive Fractional Integrated Moving Average (ARFIMA) models. Yearly guinea fowl production data for the period of 2010 to 2019 obtained from Food and Agricultural Organization (FAO-Kenya) was used in the study in which the Augmented Dickey Fuller (ADF) test was used to check for stationarity while the Hurst Exponent was used to test the long-memory property of the series. The ARIMA and ARFIMA models gave a better fit to the data and were used to forecast Guinea Fowl Weights. Fitted model forecast were evaluated via the Random Mean Squared Error (RMSE) in which the ARFIMA model was found to give a better forecast of the Guinea Fowl weights compared to the ARIMA model.
\end{abstract}

Keywords: Poultry Farming, Auto-Regression, Fractional Integration, Long-Memory, Augmented Dickey Fuller (ADF) Test, Random Mean Squared Error (RMSE)

\section{Introduction}

Food security is becoming a major concern in Kenya due to the rapid urbanization and the ever growing population of her citizens. To address this there is need to develop sound agricultural policies aimed at improving the agricultural output while at the same time preserving the environment. Poultry farming is considered as one of those sectors that need to the modernized so as to maximize output and help curb the food insecurity problem. This is due to the fact that there has been an increased demand for poultry meat in the recent past thus calling for the mechanization of the poultry farming sector.

Rural poultry production over the years has been centered on local chickens as a major contributor of protein availability for most of the rural communities. It has also supported crop agriculture where sales of chickens are made to support rural farmers' revenue input to crop production or as a cushion when revenues expected from crop production are not feasible.

Most of the birds reared in this communities are on free range production systems since not only are they affordable to these farming communities but also have fitting benefit into the cultural and social-economic lives of the associated rural farming communities. To help understand the mechanization required so as to improve the poultry farming output with regard to feed diversification, this study had a look at the rearing of guinea fowls in Kenya.

Commercial farming of guinea fowls is at its infant stages in Kenya and this type of farming is generating a lot of interest. They are raised mainly for their flesh and partly for eggs since their meat is tender and nutritious with a fine flavor similar to other game birds. It is lean and rich in essential fatty acids.

It's in this regard that this study evaluates Guinea Fowls production in Kenya with respect to their weights subject to different poultry feeds. The ARIMA and ARFIMA models were used in the study. The study thus aimed at identifying 
the order of ARIMA and ARFIMA models, estimate parameters of the models, make relevant forecasts and compare the relative forecasting performance of the fitted models so as to obtain the model that gives a better fit.

\subsection{Statement of the Problem}

With an increased demand for poultry products there is need for the market players to respond by ensuring there is an adequate supply of the same products since an increased poultry weight translates to an increased poultry mean production [4]. There has been extensive statistical research in poultry farming aimed at establishing the relationship that exist between body weight and different poultry feeds. This statistical modeling of poultry production has allowed farmers and researchers to describe and understand biological processes and prioritize the aims of production research from identifying the study components to evaluating the effect on the response variables.

This study sought to model the weight of guinea fowls subject to different types of poultry feeds namely; Horse-bean, Linseed, Soybean, Sunflower, Meat-meal and Casein using the Auto-Regressive Integrated Moving Average (ARIMA) stochastic models in comparison to the Auto-Regressive Fractional Integrated Moving Average (ARFIMA) model. This was aimed at identifying the best stochastic (ARIMA/ARFIMA) model for modeling of guinea fowl production where the guinea fowls were clustered into six groups and each group subjected exclusively to one of the feeds and the mean weight of the clusters recorded at different times.

\subsection{Objectives}

\subsubsection{General Objective}

The general objective of this study was to model the Guinea Fowls production in Kenya using the AutoRegressive Integrated Moving Average (ARIMA \& ARFIMA) models.

\subsubsection{Specific Objectives}

This study was guided by the following specific objectives;

To model guinea fowl production for different poultry feeds using the Auto- Regressive Integrated Moving Average (ARIMA \& ARFIMA) models.

To estimate the model parameters of the fitted stochastic models (ARIMA \& ARFIMA) and use them to forecast the Guinea Fowl production.

To perform the model adequacy tests of the fitted time series models (ARIMA \& ARFIMA) in modeling the Guinea Fowl production.

\subsection{Outline of the Study}

This study was centered on the use of the Auto-Regressive Integrated Moving Average methodologies (ARIMA \& ARFIMA) to model the weights of guinea fowls at different times for different poultry feeds. The subsequent sections of this study are organized as follows: chapter two for the literature review, chapter three for the study methodology, chapter four for the data analysis and chapter five for the conclusions \& recommendations.

\section{Literature Review}

\subsection{Introduction}

This chapter was established with the intention of studying previous works on poultry modeling so as to get appropriate theories and experiential proves to substantiate the study.

\subsection{Literature Review}

In the modeling of poultry production, Sankar [1] used the auto-regressive (AR), moving average (MA) and the auto-regressive integrated moving average (ARIMA) to select an appropriate stochastic model for egg production while Shakeel et al [9] modeled and forecasted Beef, Mutton, Poultry meat and total meat production using the them time series ARIMA models.

Gale \& Arnade [2] used the generalized Leontif functional form of the marginal cost to model the effect of rising chicken feeds and labor costs on chicken price while Ahmad [3] studied egg production forecasting using the general regression neural network in comparison to the linear regression predictions and the Gompertz nonlinear model.

This was as with Raji et al [10] who estimated model parameters of the Japanese quail growth curve using the Gompertz model and Semara et al [13] who compared some non-linear functions for describing broiler growth curves of Cobb500 strain using the Gompertz model.

Hanus et al, Yakubu et al, Nosike et al, Abiyu and Dzungwe et al used the linear and multiple linear regression models to study the effect of poultry linear body measurements on poultry body weight, an application to chicken and guinea fowl production [4-7, 15].

Luis et al [8] used the distributed-delay model to predict egg production in laying hens while Isife et al [11] had a design and simulated study of an automated poultry feed mixing machine using the virtual multipoint Near Infrared Spec-troscopy (NIRS) analysis with its accompanying sensor probes.

Ahmad [12] modeled poultry growth using Neural Networks and simulated data and Abdul et al [14] had a relative economic value estimation of Guinea Fowl production traits using the partial budgeting technique.

\section{Methodology}

\subsection{Introduction}

This chapter discusses the ARIMA and ARFIMA models used in the modeling of Guinea Fowls production in Kenya. A mention of the data, model diagnostics and the fitted model forecasts are also given. 


\subsection{Data}

The data for the study included monthly number of guinea fowls produced and their weight with regard to the different poultry feeds fed to them. This were be obtained from the Food Agricultural Organization (FAO) Kenya. This was for the period 2010 to 2019 .

\subsection{Time Series Modeling}

This involved the fitting of the time series models to the data. This study used the Box-Jenkins procedure to achieve this and is defined as; model identification, model estimation, model verification, test for the white noise and then forecasting of the fitted models. The Auto-Regressive Integrated Moving Average (ARIMA) and the Auto-Regressive Fractional Integrated Moving Average (ARFIMA) models were used in the modeling of the Guinea Fowl Production.

\subsubsection{Stationarity Test}

Prior to the fitting of the Auto-Regressive models to the Guinea Fowls Production data, the data was tested for stationarity using the Augmented Dickey-Fuller (ADF) test. By letting $\phi$ to be the coefficient of the lagged dependent variable (Guinea Fowl Weights), the test statistic is given as;

$$
A D F=\frac{\widehat{\phi}}{S E(\widehat{\phi})}
$$

\subsubsection{Long Memory Test}

The Hurst Exponent produced by re-scaled range analysis was used to test for the long memory in the series. This was by first estimating the range $(\mathrm{R})$ for a given period $(t)$ of the series as;

$$
R_{t}=\max \sum_{j=1}^{t}\left(Y_{j}-\bar{Y}\right)-\max \sum_{j=1}^{t}\left(Y_{j}-\bar{Y}\right)
$$

where $\bar{Y}$ is the overall mean of the series. Letting $S_{t}$ to be the standard deviation of $Y_{t}$, we have the Hurst exponent as;

$$
H=\frac{\log \frac{R_{t}}{S_{t}}-\log \alpha}{\log t}
$$

When $0<\mathrm{H}<1$ there exists a long-memory structure in the series.

\subsubsection{The Auto-Regressive Integrated Moving Average Models}

In order to model the time series data (Guinea Fowl Weights Production Data), the ARIMA and ARFIMA models were fit to the data.

The general expression of the ARIMA process $y_{t}$ is defined by the equation;

$$
\left(1-\Phi_{p} B\right) \nabla^{d} y_{t}=\left(1-\Theta_{q} B\right) e_{t}
$$

Where $\left(1-\Phi_{p} B\right) \nabla^{d} y_{t}=e_{t}$ is the Auto-Regressive component (AR) and $y_{t}=\left(1-\Theta_{q} B\right) \nabla^{d} e_{t}$ is the Moving-Average (MA) component.

In the case of the integration parameter $d$ being fractional and greater than zero, the ARIMA model exhibits a long memory process and is thus referred to as the ARFIMA model. The general expression of the ARFIMA process $y_{t}$ is defined by the equation;

$$
\Phi(B) y_{t}=\Theta(B)(1-B)^{-d} e_{t}
$$

Where $\Phi(B)=1-\Phi_{1} B-\cdots-\Phi_{P} B^{p}$ and $\Theta(B)=1+$ $\Theta_{1} B+\cdots+\Theta_{q} B^{q}$ are the AR and MA terms respectively.

$B$ is the backward shift operator and $(1-B)^{-d}$ is the fractional differencing operator given by the binomial expression;

$$
(1-B)^{-d}=\sum_{j=0}^{\infty} \frac{\Gamma(j+d)}{\Gamma(j+1) \Gamma(\mathrm{d})} B^{j}=\sum_{j=0}^{\infty} n_{j} B_{j}
$$

\subsection{Parameter Estimation}

For the Integrated Time Series models, we define a special case of the models in which $\Theta(B)=\Phi(B)=1$ with a spectral density given as;

$$
f(\lambda)=\frac{\sigma^{2}}{2 \pi} \int_{-\pi}^{\pi}\left|1-e^{-i \lambda}\right|^{-2 d}
$$

Parameter estimation was via the maximum likelihood estimation technique and the associated log-likelihood was given as;

$$
L(\Omega) \approx-\frac{1}{4 \pi}\left[\int_{-\pi}^{\pi} \log [2 \pi f(\lambda)] d \lambda+\int_{-\pi}^{\pi} \frac{I(\lambda)}{f(\lambda)} d \lambda\right]
$$

where $I(\lambda)=\sum_{j=1}^{n} \frac{\left|y_{j} e^{i \lambda j}\right|^{2}}{2 \pi n}$ is the periodogram.

\subsection{Order Determination and Model Diagnostics}

Order determination for the fitted models was via the AIC $\&$ BIC information criterion. Goodness of fit tests for the fitted models was via the Ljung Box-Test, Standardized Residuals, Random Mean Squared Error and the analysis of residual auto-correlation functions (ACFs) and partial auto-correlation functions (PACFs).

\section{Results and Discussions}

\subsection{Introduction}

This chapter is established on the premises of giving a data analysis for the Auto- Regressive Integrated Moving Average (ARIMA) and Auto- Regressive Fractional Integrated Moving Average (ARFIMA) Models in modeling poultry feed effect on Guinea fowl production in Kenya.

\subsection{Exploratory Data Analysis}

To aid in the preliminary exploration of the data, a total of 87 observations on the weights of Guinea Fowls was used in the study. Newly hatched Guinea Fowls were randomly allocated into six groups and each group given a different feed supplements (horse-bean, linseed, soybean, sunflower, meat meal and casein) in which their weights after Four weeks were recorded in grams. 


\subsubsection{Descriptive Statistics of the Data}

Table 1 gave the descriptive statistics of the Guinea Fowl weights in which the mean weight was estimated at 286.6 grams with a median of 295 grams. The maximum and minimum weights were 439 grams and 108 grams respectively. The first and third quartiles were 219.5 grams and 364.0 grams respectively.

The mean Guinea Fowl weights were smaller than the median weights which gave an indication of majority of the weights being to the right of the mean value. This was attributed to the continuous increase in weights by the Guinea Fowls over time as confirmed by a higher $3^{\text {rd }}$ Quartile compared to the $1^{\text {st }}$ Quartile of the Guinea Fowl Weights.

Table 1. Guinea Fowl Weights Descriptive Statistics.

\begin{tabular}{llllll}
\hline Min & $\mathbf{1}^{\text {st }} \mathbf{Q}$ & Med & Mean & $\mathbf{3}^{\text {rd }} \mathbf{Q}$ & Max \\
\hline 108.0 & 219.5 & 295.0 & 286.6 & 364.0 & 439.0 \\
\hline
\end{tabular}

\subsubsection{Stationarity Test}

In order to identify the presence of stationarity in the Guinea Fowl production data, the time plot and the Dickey-Fuller test were used. Table 2 gave the Dickey-Fuller test.

Table 2. Dickey-Fuller Test for Stationarity.

\begin{tabular}{lll}
\hline Dickey-Fuller & Lag-Order & P-Value \\
\hline-4.1521 & 4 & 0.01 \\
\hline
\end{tabular}

The Dickey-Fuller p-value was small at a -4.1524 test statistic and an additional lag of 4 . This gave an indication of the presence of stationarity in the data after a first difference thus ascertained the fitting of the integrated time series models to the data.

\subsubsection{Test for Correlation}

The auto-correlation and partial auto-correlation functions of the Guinea Fowl Weights Data were given as in Figure 1. The auto-correlation function was used to show how correlated weights are with each other based on how many steps they were separated by.

For the Guinea Fowl production data, the auto-correlation functions (ACFs) crossed the blue dashed line which gave an indication of the presence of correlation among the Guinea Fowl Weights. The ACFs had a slow exponential decay which was evident as their magnitude became smaller with increase in the time lag giving a further indication of the presence of non-stationarity in the data.

The partial auto-correlation functions (PACFs) were used to show the degree of association between the Guinea Fowl weights and time while adjusting the effect of the poultry feeds. From Figure 1, the PACFs showed a specific pattern which did not repeat thus a conclusion of the absence of seasonality in the data. This suggested that ARIMA and ARFIMA models would be appropriate for the series.
Time Series Data ACF

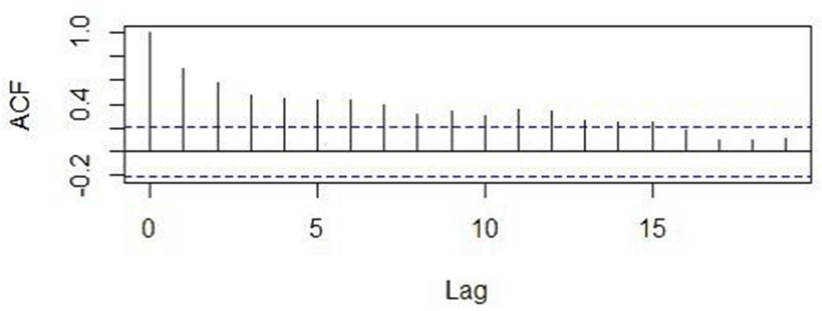

Time Series Data PACF

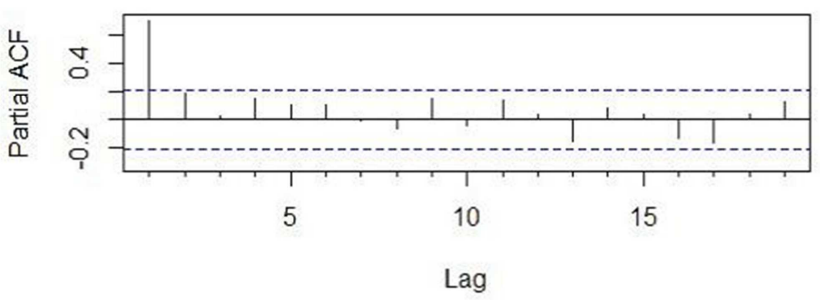

Figure 1. ACF and PACF of the Guinea Fowl Weights.

\subsection{ARIMA Modeling}

Since the study proved the Guinea Fowls Production data to be a non-stationanary series that becomes stationary after first difference, the ARIMA models were fit to the data. On fitting the ARIMA model to the data, the following feasible tentative models were compared; $\operatorname{ARIMA}(0,1,1)$, ARIMA $(1,1,1), \operatorname{ARIMA}(0,1,2), \operatorname{ARIMA}(1,1,0), \operatorname{ARIMA}(1,1,2)$ and ARIMA $(0,1,0)$. Table 3 gave the fitted ARIMA models to the Guinea Fowl Weights data and their corresponding AIC/BIC.

The ARIMA $(1,1,1)$ had the lowest AIC/BIC values followed by ARIMA $(0,1,2), \operatorname{ARIMA}(1,1,2), \operatorname{ARIMA}(0,1$, $1), \operatorname{ARIMA}(1,1,0)$ and lastly the $\operatorname{ARIMA}(0,1,0)$ had the highest AIC/BIC values. This made the ARIMA $(1,1,1)$ best model for modeling Guinea Fowl Production data since it had the lowest AIC and BIC values of 763.6626 and 770.2211 respectively.

Table 3. AIC \& BIC of Fitted ARIMA Models.

\begin{tabular}{lll}
\hline ARIMA MODEL & BIC & AIC \\
\hline ARIMA $(0,1,1)$ & 770.2686 & 765.8296 \\
ARIMA $(1,1,1)$ & 770.2211 & 763.6626 \\
ARIMA $(0,1,2)$ & 770.0949 & 764.2363 \\
ARIMA $(1,1,0)$ & 777.3611 & 772.9221 \\
ARIMA $(1,1,2)$ & 769.5397 & 765.6616 \\
ARIMA $(0,1,0)$ & 782.0691 & 779.8496 \\
\hline
\end{tabular}

Table 4 gave the parameter estimation of the $\operatorname{ARIMA}(1,1$, 1) model of order one with its corresponding coefficients and standard error. The auto-regressive component of the model was estimated at 0.2940 with a standard error of 0.1393 while the moving average component of the model was estimated at -0.8701 with a standard error of 0.0636 . The variation in the fitted model was estimated at 4188 with a log-likelihood of -379.9. This were the parameter estimates of the model with the smallest AIC after the first difference. 
Table 4. Fitted ARIMA Model Coefficients.

\begin{tabular}{lll}
\hline & AR (1) & MA (1) \\
\hline Coefficient & 0.2940 & -0.8701 \\
Std Error & 0.1393 & 0.0636 \\
\hline
\end{tabular}

\subsection{ARFIMA Modeling}

In order to model the long-memory component of the Guinea Fowl Production Time Series, the ARFIMA models were fit to the data. Since this series becomes stationary after the first difference, the ARFIMA $(1,1,1)$ model was fit to the data and its model coefficients given as in Table 5 .

Table 5. Fitted ARFIMA Model Coefficients.

\begin{tabular}{lllll}
\hline & Estimate & Std. Error & Z-Value & $\operatorname{Pr}(>|\mathbf{z}|)$ \\
\hline Phi & 0.9993 & $6.33 \times 10^{-7}$ & $1.58 \times 10^{6}$ & $2.22 \times 10^{-16}$ \\
Theta & 0.9186 & $7.81 \times 10^{-2}$ & 11.7670 & $2.22 \times 10^{-16}$ \\
H & 0.7223 & 0.1353 & 5.3396 & $9.31 \times 10^{-8}$ \\
Set. Mean & 1000 & NA & NA & NA \\
\hline
\end{tabular}

The moving average component of the model was estimated at 0.9993 while that of the auto-regressive component was estimated at 0.9186. The Hurst-Exponent was estimated at 0.7223 which gave an indication of the presence of the long-memory property in the data thus necessitating the need of fitting the ARFIMA models to the data. The set mean of the Guinea Fowls weights was at 1000 grams.

\subsection{Fitted Model Diagnostics}

In order to check on the goodness of fit of the fitted ARIMA $(0,1,1)$ model in the modeling of Guinea Fowls Weights data, the fitted model residuals were analyzed. The standardized residuals, ACF of residuals and the Ljung Box $\mathrm{P}$-Values were used to aid in the model residual analyses.

To test for auto-correlations in the residuals, Table 6 gave a summary of the Ljung Statistic. The Ljung Statistic p-value was more than 0.5 which gave an indication of the absence of auto-correlations in the residuals. The residuals were thus not distinguishable from a white noise series hence the notion that the ARIMA $(1,1,1)$ fitted the Guinea Fowl Weights data well.

Table 6. The Ljung Statistic.

\begin{tabular}{lll}
\hline X-Squared & Df & P-Value \\
\hline 0.090744 & 1 & 0.7632 \\
\hline
\end{tabular}

Figure 2 gave the fitted model diagnostic plots for residual ACF and PACF of the fitted ARIMA $(1,1,1)$ model. The model verification was concerned with checking the residuals of the fitted model to see if they contained any systematic pattern which still could be removed to improve the chosen ARIMA. This has been done through examining the autocorrelation and partial auto-correlation of the residuals.

The plots showed that there were no serial correlation observed in the residuals of the series and were within the tolerance line in the p-value plot with a mean close to zero. The ACF of the residuals do not exceed a significant bound from lag 1 to the end thus the indication that the fitted model was adequate and good in the modeling of Guinea Fowl Production Time Series data.
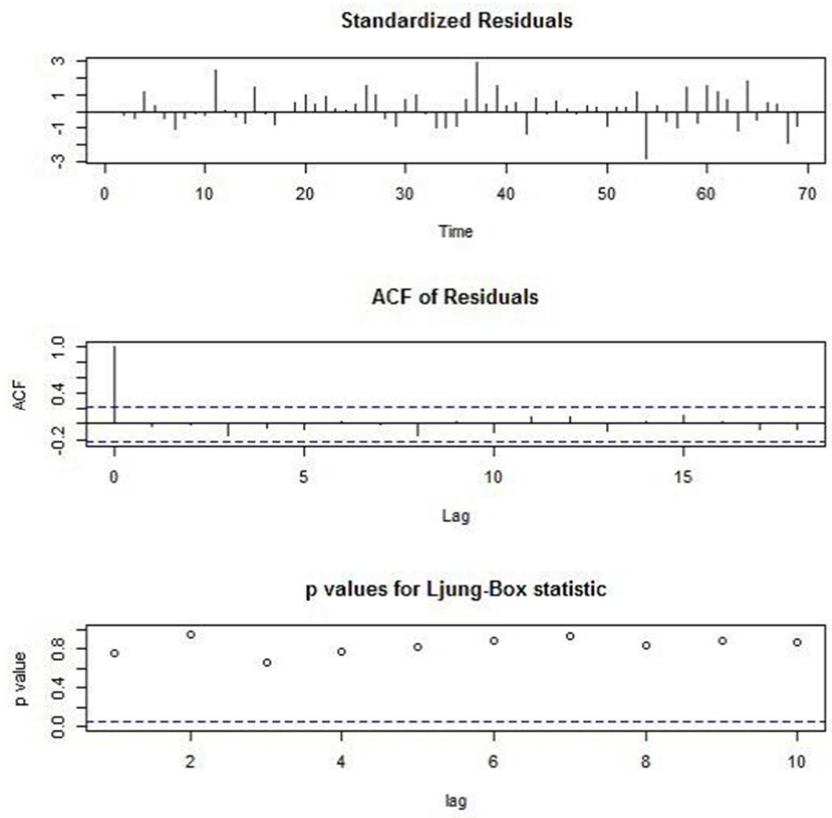

Figure 2. Guinea Fowl Weights Diagnostic Plot.

Figure 3 gave a histogram of the fitted model residuals which gave an indication of the residuals being normally distributed. The normality distribution of the fitted model residuals also gave an indication of a better fit of the ARIMA $(1,1,1)$ in the modeling of Guinea Fowl Weight Production Data.

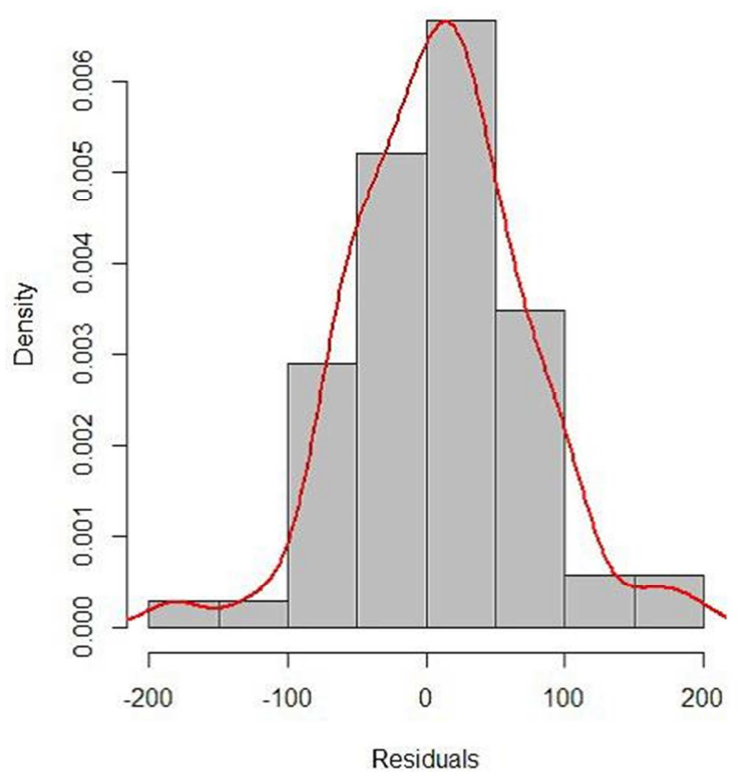

Figure 3. Histogram of Residuals.

Figures 2 and 3 graphs gave an indication that the fitted model captured the data well (it accounted for all the available information) and therefore can be used to predict future Guinea Fowl Weights. 


\subsection{Guinea Fowls Weight Forecasting}

The fitted ARIMA $(1,1,1)$ and $\operatorname{ARFIMA}(1,1,1)$ were used to forecast future Guinea Fowl Weights over time. Figures 4 and 5 gave a visualization of the Guinea Fowl Weight Forecast for the fitted models. The training range data was used to do model forecasting. The model forecast was at the beginning of the forecast range and is given by the straight line. The confidence intervals for the training range data and the model forecast are also provided.

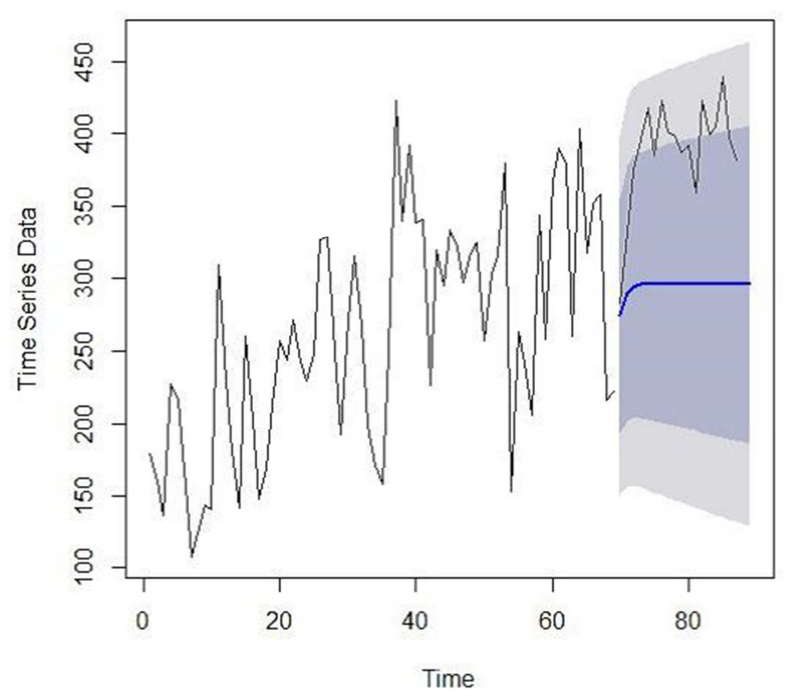

Figure 4. ARIMA Guinea Fowl Weights Forecasted Plot.

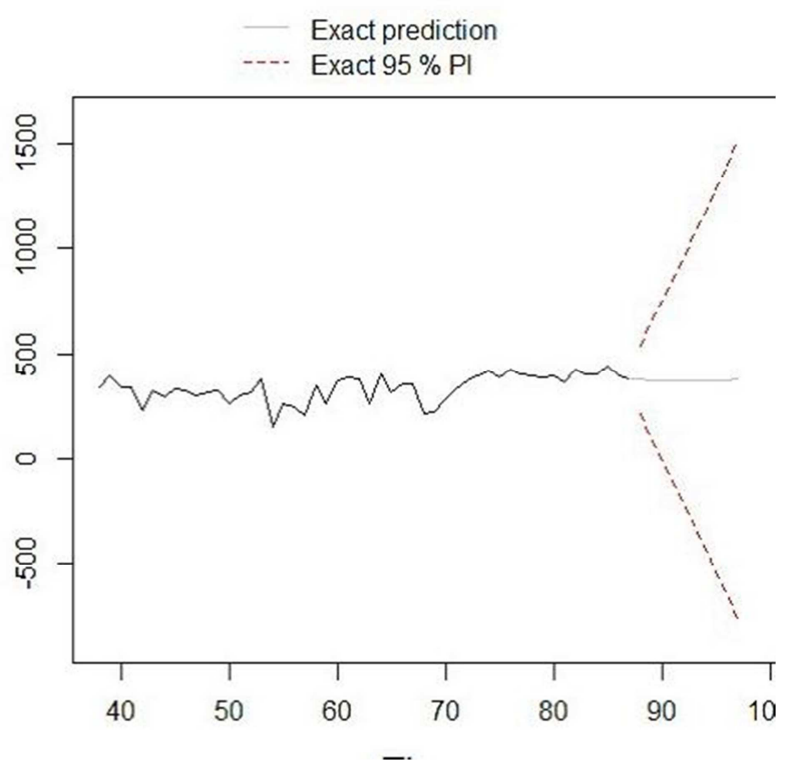

Figure 5. ARFIMA Guinea Fowl Weights Forecasted Plot.

The fitted model forecasts were estimated at 95\% confidence intervals. Since the constant $\mathrm{c}$ was not equal to 0 and $\mathrm{d}$ was equal to 1 , the long term forecast followed a straight line as in Figures 4 and 5. From the model forecasts, the prediction intervals increased in size within the forecast horizon. Forecast calculations assumed that the residuals are uncorrelated and normally distributed.
In order to identify a more parsimonious model between the two fitted models, we finally compare the forecast values of ARFIMA $(1,1,1)$ and $\operatorname{ARIMA}(1,1,1)$ with the observed values. To evaluate their performance, the Root Mean Squared Error (RMSE) was used. The ARIMA model had an RMSE of 0.6268 while the ARFIMA model had an RMSE of 0.4868 . From this results the RMSE value of ARFIMA was smaller than the RMSE value of ARIMA model thus the conclusion that ARFIMA model is a much better model than the ARIMA model in the modeling of Guinea Fowl production data.

\section{Conclusions and Recommendations}

\subsection{Introduction}

This is the final chapter of the study and it gives the conclusion and recommendations of the study with regard to the modeling of Guinea Fowls Weights Production data.

\subsection{Conclusion}

In the modeling of Guinea Fowl Production data, the study gave an application of the integrated ARIMA and ARFIMA models in which both models fitted the data perfectly well. Even though both models gave a better fit to the data, forecasts obtained using the ARFIMA model were closer to the actual values than forecasts obtained using ARIMA model as evidenced by the Root Mean Squared Error (RMSE). The ARFIMA model had a lower RMSE which showed that it gave better forecasts than the ARIMA model. This gave the conclusion that in the modeling of Guinea Fowls Weights over time, the ARFIMA model is a better model than the ARIMA model.

This is as with Sankar [1] and Shakeel et al [9] who stipulated the need for a time series analysis of poultry production so as to capture historical traits that influence the production of poultry products. On the need to capture the long memory property of a time series, Raji et al [10], Semara et al [13] and Ahmad [12] recommended the use of a long process to model the same which gave a motivation for the study. This in turn helped model the long-term correlations of the poultry production data using the Auto-Regressive Fractional Integrated Moving Average models.

In conclusion, Guinea Fowl production weights have a positive increase over time. Hence, little more effort would be required by poultry farmers in order to meet up with increasing demand for the products due to increase in population. Moreover, the Autoregressive Integrated Moving Average (ARIMA AND ARFIMA) time series models are effective tools in examining Guinea Fowl production weights and should be employed at regular intervals to monitor the production rate.

\subsection{Recommendations}

This study gave an application of modeling Guinea Fowl Weights using the Integrated and the Fractionally 
Integrated Time Series models in the modeling of Guinea Fowl Production data. The study further acknowledges the need to expound on this work by future researching on the multivariate modeling of the long-memory models for simulated data and incorporating their Bayesian estimates.

\section{Acknowledgements}

I thank God for giving me the perseverance and endurance to go through my research project. I would like to acknowledge with gratitude, my supervisors, Dr. Thomas Mageto and Dr. Anthony Wanjoya. Thank you so much for putting up with me during this process, reading my many drafts and still encouraging me up to this far. Special thanks to my fellow colleagues, Evan Kithure and Lucy Pembe for fueling my writing and being generous with some points in my study. I extend my deepest and warmest appreciation to my husband, Nick and children, Sackey and Gideon for their encouragement to complete my study.

\section{References}

[1] Sankar, T. J. (2014). Design of a Stochastic Forecasting Model for Egg Production. International Journal of Innovative Science, Engineering \& Technology, S1 (6).

[2] GALE, F. \& Arnade, C. (2015). Effects of Rising Feed and Labor Costs on China's Chicken Price. International Food and Agribusiness Management Review, 18 (A).

[3] Ahmad, H. A. (2012). Egg production forecasting: Determining efficient modeling approaches. Journal of Applied Poultry and Research, 20 (4); 463-473. doi: 10.3382/japr.2010-00266.

[4] Hanus, A., Hanusoval, E., Oravcova, M. \& Hrncar, C. (2017). Factors affecting growth in Oravka chicken breed. Slovak J. Anim. Sci., 50 (3); 112-117.

[5] Yakubu, A., Oluremi, O. A. \& Ibrahim, Z. N. (2018). Modelling egg production in Sasso dual-purpose birds using linear, quadratic, artificial neural network and classification regression tree methods in the tropics. Livestock Research for Rural Development 30 (10).
[6] Nosike, R. J., Okoro, V. M. O. \& Ukwu, H. O. (2016). Statistical Modelling of Body Weight and Linear Body Measurements in Nigerian Indigenous Chicken. Journal of Agriculture and Veterinary Science, 7 (1); 27-30.

[7] Abiyu, T. (2019). Statistical Modelling of Indigenous Chicken with Body Weight and Linear Body Measurements in Bench Maji Zone, South Western Ethiopia. International Journal of Environmental Sciences and Natural Resources, 22 (2). doi: 10.19080/IJESNR.2019.22.556083.

[8] Luis, G. V., Mario, C. M., Daniel, R. \& Jose, M. C. (2013). Using the distributed-delay model to predict egg production in laying hens. Rev Colomb Cienc Pecu, 2013 (26); 270-279.

[9] Shakeel, N. \& Masood, A. K. (2014). Modeling and Forecasting of Beef, Mutton, Poultry Meat and Total Meat Production of Pakistan for Year 2020 by using Time Series ARIMA Models. European Scientific Journal, 3 (special issue).

[10] Raji, A. O., Alade, N. K. \& Duwa, H. (2014). Estimation of Model Parameters of the Japanese Quail Growth Curve using Gompertz Model. Arch. Zootec. 63 (243); 429-435.

[11] Isife, J. K., Ukwani, C. \& Sani, G. (2019). Design and Simulation of an Automated Poultry Feed Mixing Machine Using Process Controller. Global Scientific Journals, 7 (1); 537-602.

[12] Ahmad, H. A. (2019). Poultry Growth Modeling using Neural Networks and Simulated data. Journal of Applied Poultry Research, 18; 440-446. doi: 10.3382/japr.2008-00064.

[13] Semara, L., Mouffok, C. \& Belkasmi, F. (2019). Comparison of some Non-Linear Functions for Describing Broiler Growth Curves of Cobb500 Strain. Poultry Science Journal, 7 (1); 51-61. doi: 10.22069/psj.2019.15965.1386.

[14] Abdul, S. M., Bonsu, F. R. K., Abunyuwah, I. \& Serekye, A. Y. (2020). Relative Economic Value Estimates of Guinea Fowls (Numida meleagris) Production Traits. World Journal of Advanced Research and Reviews, 7 (1); 273-281. doi: 10.30574/wjarr.2020.7.1.0265.

[15] Dzungwe, J. T., Gwaza, D. S. \& Egahi, J. O. (2018). Statistical Modeling of Body Weight and Body Linear Measurements of the French Broiler Guinea Fowl in the Humid Tropics of Nigeria. Poultry, Fisheries and Wildlife Sciences, 6 (2); 1-4. doi: 10.4172/2375-446X. 1000197. 